\title{
Elemental abundance ratio comparisons of globular clusters, field stars, and dwarf spheroidal galaxies
}

\author{
Barton J. Pritzl ${ }^{1}$, Kim A. Venn ${ }^{1}$, \\ and Mike J. Irwin ${ }^{2}$ \\ ${ }^{1}$ Department of Physics and Astronomy, Macalester College, 1600 Grand Ave., Saint Paul, MN \\ 55105, USA \\ email: pritzl@macalester.edu, venn@macalester.edu \\ ${ }^{2}$ Institute of Astronomy, Madingley Road, Cambridge CB3 0HA, UK \\ email: mike@ast.cam.ac.uk
}

\begin{abstract}
We have compiled a sample of globular clusters with high-resolution abundances from the literature to compare to the chemistries of stars in the Galaxy and those in dwarf spheroidal galaxies using the $[\alpha / \mathrm{Fe}]$ and light r-process element ratios. From existing kinematic data we are able to analyze the populations according to their Galactic components (bulge, thin disk, thick disk, and halo). We find that most globular clusters mimic the Galactic field population arguing for a similar chemical evolution history. Possible extragalactic globular clusters are also noted.
\end{abstract}

Keywords. Galaxy: abundances - galaxies: abundances - globular clusters: general - stars: abundances

\section{Abundance Ratios}

Detailed elemental abundances were found for 45 globular clusters within the past 20 years. To standardize the different datasets, abundance ratios were corrected to the solar abundances and $\log g f$ values from the Lick-Texas group, when possible. The Galactic components for 29 globular clusters were determined from a calculation of their phase space using kinematic data from Dinescu (2004, private communications), while the components for the remaining globular clusters were based solely on their positional vector (see Fig. 1, left panel). All clusters with $R<2.7 \mathrm{kpc}$ are classified as bulge clusters. Further details can be found in Pritzl, Venn, \& Irwin (2005). In general, the majority of Galactic globular clusters follow the abundance trends of the Galactic field stars for both the $\alpha$-elements (Mg, Si, Ca, \& Ti) and the neutron-capture elements (Y, Ba, La, \& Eu). This implies a similar chemical evolution throughout the Galaxy (see also the review by C. Sneden in this proceeding). However, Palomar 12 and Ruprecht 106 have noticably lower $[\alpha / \mathrm{Fe}]$ ratios, and Terzan 7 is also slightly lower. This is not unexpected since dwarf spheroidal galaxies are known to have subsolar $[\alpha / \mathrm{Fe}]$ abundance ratios (Venn et al. 2004) and all three clusters are thought to have extragalactic origins (Ibata, Gilmore, \& Irwin 1994; Lin \& Richer 1992). M68 is a possible extragalactic globular cluster given its slightly younger age compared to globular clusters of similar metallicity and its high Galactocentric radial velocity. 

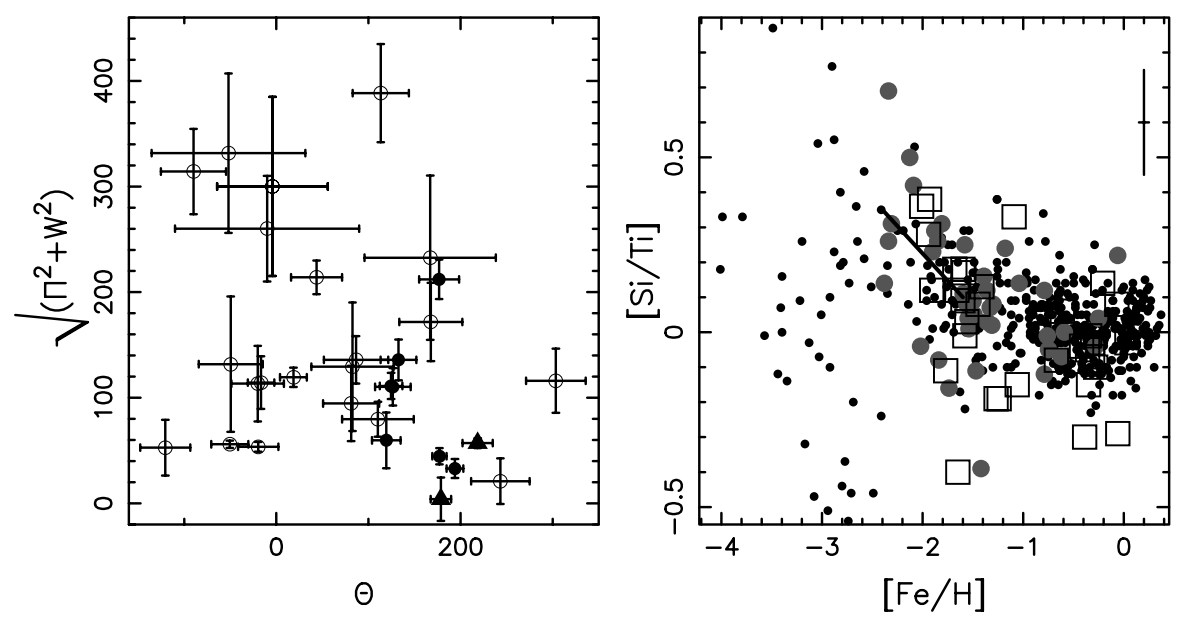

Figure 1. Left panel: Galactic component allocations for globular clusters (open circle - halo, filled circle - thick disk, triangle - thin disk) from Pritzl et al. (2005). Right panel: [Si/Ti] in globular clusters, and Galactic and dwarf spheroidal field stars. The solid line is a representation of the apparent decreas in the ratio at intermediate metallicities (see text).

\section{2. $[\mathrm{Si} / \mathrm{Ti}]$ Trends}

One interesting abundance ratio is $[\mathrm{Si} / \mathrm{Ti}]$ for the globular clusters. Lee \& Carney (2002) found that there appears to be a trend of decreasing [Si/Ti] with increasing Galactocentric distance for the "old" halo globular clusters. Using our database, no significant trend of $[\mathrm{Si} / \mathrm{Ti}]$ decreasing with apogalactic or Galactocentric distance was detected for "old" halo globular clusters as classified by (Mackey \& Gilmore 2004). However, it was found that all the clusters with high $[\mathrm{Si} / \mathrm{Ti}]$ ratios were also metal-poor $([\mathrm{Fe} / \mathrm{H}]<-1.9)$. In Figure 1 (right panel), [Si/Ti] is plotted as a function of $[\mathrm{Fe} / \mathrm{H}]$ for the globular clusters along with Galactic and dwarf spheroidal field stars. The scatter seen below $[\mathrm{Fe} / \mathrm{H}]<-2.4$ may be attributed to inhomogeneous mixing in the Galaxy. From $[\mathrm{Fe} / \mathrm{H}]=-2.4$ to about -1.6 , the $[\mathrm{Si} / \mathrm{Ti}]$ ratio begins at an elevated level $(\sim+0.5)$ and steadily decreases until it levels off. The high ratios of [Si/Ti] may be explained by massive stars $\left(M>30 M_{\odot}\right)$ which produce higher amounts of Si compared to Ti (Woosley \& Weaver 1995). An increasing number of less massive stars contributing to the interstellar medium would decrease the $[\mathrm{Si} / \mathrm{Ti}]$ ratio, if thoroughly mixed. It is interesting to see that a number of dwarf spheroidal stars also show lower [Si/Ti] ratios than the mean, which could be related to selective "blow-out" of massive star yields or a truncated initial mass function.

\section{Acknowledgements}

This research is supported by a CAREER award (AST 99-84073) from the National Science Foundation.

\section{References}

Ibata, R.A., Gilmore, G., \& Irwin, M.J. 1994, Nature 370, 194

Lee, J.-W., \& Carney, B.W. 2002, AJ 124, 1511

Lin, D.N.C., \& Richer, H.B. 1992, ApJ 388, L57

Mackey, A.D., \& Gilmore, G.F. 2004, MNRAS 355, 504

Pritzl, B.J., Venn, K.A., \& Irwin, M.J. $2005 A J$ in press, astro-ph()

Venn, K.A., Irwin, M., Shetrone, M.D., Tout, C.A., Hill, V., \& Tolstoy, E. 2004, AJ 128, 1177

Woosley, S.E., \& Weaver, T.A. 1995, ApJS 101, 181 